\title{
Crescimento do curauá branco sob efeito de subdoses de glyphosate
}

\author{
Ananas erectifolius Growth Under Effect of Glyphosate Low Doses \\ Cleber Daniel de Goes Maciel ${ }^{1}$; Edivaldo Domingues Velini ${ }^{2}$; Renan Florentino dos Santos ${ }^{3}$; \\ Antônio Giovanni Pontes Viana ${ }^{4}$
}

\begin{abstract}
Resumo - Planta nativa da Amazônia, o curauá (Ananas erectifolius) tem sido uma espécie de grande interesse para indústria automobilística, devido às fibras de suas folhas apresentarem resistência, maciez e peso reduzido. Alguns estudos sobre simulação de deriva de glyphosate têm demonstrado a ocorrência de tolerância e/ou estímulos de crescimento da parte aérea de algumas espécies vegetais. Desta forma, com o objetivo de avaliar os efeitos da aplicação de subdoses de glyphosate no crescimento, desenvolvimento e produtividade de curauá branco um experimento foi conduzido a campo na Fazenda Pematec Triangel do Brasil Ltda, Santarém/PA. Os tratamentos estudados foram constituídos pela aplicação de glyphosate nas concentrações de 0,0 ; 11,$2 ; 22,5 ; 45,0 ; 90,0 ; 180,0 ; 360,0$ g e.a. ha ${ }^{-1}$ e uma testemunha capinada. As plantas de curauá branco apresentaram baixa tolerância ao herbicida glyphosate, sendo que subdoses superiores a 45,0 g e.a. ha ${ }^{-1}$ promoveram as reduções mais significativas de produtividade de folhas beneficiáveis quando aplicadas em pós-emergência. Nenhuma das subdoses estudadas promoveu estimulo ou incremento do desenvolvimento vegetativo das plantas de curauá branco.
\end{abstract}

Palavras-chave: Ananas erectifolius, seletividade, desenvolvimento, produtividade.

\begin{abstract}
Amazonian native plant, Ananas erectifolius has been a specie of great interest for automobile industry, due to the fibers contained in its leaves that show resistance, softness and reduced weight. Some studies about simulation of glyphosate drift have been demonstrating the occurrence of tolerance and/or incentives of dossel growth of some vegetable species. This way, with the objective of evaluating the effects of glyphosate subdoses application in growth, development and yield of Ananas erectifolius an experiment was conducted in field conditions at Fazenda Pematec Triangel do Brasil Ltda, Santarém County, PA State. Studied treatments were constituted by glyphosate application in the concentrations of $0.0 ; 11.2 ; 22.5 ; 45.0 ; 90.0 ; 180.0$; $360.0 \mathrm{~g}$ a.e. $\mathrm{ha}^{-1}$ and a hoe check. Ananas erectifolius plants presented low tolerance to glyphosate herbicide, and superior subdoses to $45.0 \mathrm{~g}$ a.e. ha $^{-1}$ promoted the most significant reductions of benefitted leaves yield when applied in post-emergency. None of the studied subdoses promoted incentive or increase of Ananas erectifolius plants vegetative development.

\footnotetext{
${ }^{1}$ Prof. Dr., Depto de Fitotecnia. Escola Superior de Agronomia de Paraguaçu Paulista - ESAPP/FUNGE. Paraguaçu Paulista-SP, e-mail: macielconsultoria@fca.unesp.br

${ }^{2}$ Prof. Dr., Depto. Agricultura FCA/Unesp, Fazenda Experimental Lageado, Caixa Postal 237, Botucatu-SP, 18603970.

${ }^{3}$ Eng. Agr. PEMATEC Triangel do Brasil Ltda, Santarém-PA.

${ }^{4}$ Tec. Agr. PEMATEC Triangel do Brasil Ltda, Santarém-PA.
}

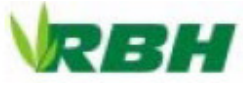


Key-words: Ananas erectifolius, selectivity, development, yield.

\section{Introdução}

O curauá (Ananas erectifolius L. B. Smith) é uma bromeliacea nativa da flora Amazônica de grande interesse comercial devido sua multiplicidade de aplicação, e principalmente como produtora de fibra de excelente qualidade utilizada na indústria automobilística, devido sua resistência, maciez e peso reduzido.

Do ponto de vista tecnológico, o emprego do curauá para a produção de compósitos poliméricos e mantas não-tecido visa melhor aproveitamento desta fibra natural lignocelulósica, além de fornecer novas alternativas de grande importância tecnológica na utilização da biomassa vegetal na produção de materiais mais compatíveis com o ambiente (Caraschi \& Leão, 2000). Para a indústria, as principais características da fibra do curauá são: resistência mecânica; leveza; ausência de odor; suavidade ao toque; facilidade de composição com outras fibras; vasta gama de utilização na indústria automotiva; possibilidade de utilização em outros produtos/mercados, como têxtil (fios e tecidos), plástico (reforço substituto a fibra de vidro), farmacêutico (anestésico e antibactericidas) e papel e celulose (papéis especiais).

A presença das plantas daninhas tem sido considerada um dos maiores problemas na manutenção do cultivo do curauá em escala comercial. Entre as justificativas estão os prejuízos ao crescimento, causados pela competição por luz, nutrientes e água, e da interferência de natureza alelopática (Pitelli, 1987). Em contra partida, o herbicida glyphosate é utilizados nos mais diferentes sistemas agrícolas para o controle de plantas daninhas, e é classificado como não seletivo, promovendo sintomas de fitointoxicação em plantas como cloroses foliares, as quais evoluem para necroses, murchas e enrolamento das folhas, superbrotação e morte (Vidal, 1997; Trezzi et al., 2001; Rodrigues \& Almeida, 2005). Entretanto, estudos com subdoses de glyphosate simulando deriva desenvolvidos por Magalhães et al. (2001a; 2001b) demonstraram que altas concentrações (12\% de $1.440 \mathrm{~g}$ e.a. $\mathrm{ha}^{-1}$ ) afetaram o desenvolvimento das plantas de milho e sorgo, ao contrário de baixas concentrações ( $2 \%$ a $4 \%$ ), onde não foi afetado o desenvolvimento e a produtividade do milho.

Alguns autores mencionam que baixas doses de glyphosate podem estimular o crescimento de plantas, sendo este efeito denominado "hormesis". (Duke et al., 2006; Cedergreen et al., 2007; Velini et al., 2010). Velini et al. (2008) observaram estímulos de crescimento da parte aérea de soja (não transgênica e susceptível ao glyphosate), eucalipto, milho, pinus, milho, café e citros, para doses de glyphosate inferiores a $36 \mathrm{~g}$ e.a.ha ${ }^{-1}$, aplicadas em simulação de deriva. De forma semelhante, Cedergreen (2008) mencionaram que os herbicidas glyphosate e metsulfuron-methyl podem induzir o crescimento da biomassa de plantas de cevada em aproximadamente $25 \%$, quando aplicados em doses de 5-10\% da dose comercial. Schabenberger et. al (1999) também encontraram estimulo em uma forrageira, assim como Wagner et al (2003) e Neves et al. (2009) em plantas de milho e algodoeiro.

Desta forma, o trabalho teve como objetivo avaliar os efeitos da aplicação de subdoses de glyphosate no desenvolvimento de plantas de curauá branco, assim como os reflexos das mesmas sobre o controle inicial das plantas daninhas.

\section{Material e métodos}

O trabalho foi conduzido no campo, em área de produção comercial da Fazenda 
Pematec Triangel Ltda, localizada no Município de Santarém - PA, utilizando-se a espécie curauá branco (Ananas erectifolius), espaçamento de $0,4 \mathrm{~m}$ entre linhas e plantas, e solo com características físicas de $461 \mathrm{~g} / \mathrm{kg}$ de areia; $458 \mathrm{~g} / \mathrm{kg}$ de argila e $81 \mathrm{~g} / \mathrm{kg}$ de silte.

$\mathrm{O}$ delineamento experimental utilizado foi o de blocos casualizados (DBC) com 8 tratamentos e 5 repetições, sendo as unidades experimentais constituídas por parcelas de 3,0 x 2,0 m $\left(6,0 \mathrm{~m}^{2}\right)$ com cinco linhas de plantas de curauá. Os tratamentos foram representados pelas aplicações do herbicida glyphosate (Roundup Original ${ }^{\circledR}$ ), nas concentrações de 0,$0 ; 11,2 ; 22,5 ; 45,0 ; 90,0 ; 180,0 ; 360,0$ g e.a. $\mathrm{ha}^{-1}$ e uma testemunha capinada.

A aplicação dos tratamentos foi realizada em pós-emergência do curauá e das plantas daninhas em 27/06/2006, utilizando-se um pulverizador costal, pressurizado constantemente com $\mathrm{CO}_{2}$, na pressão de trabalho de $210 \mathrm{kPa}$, equipado com barra de quatro pontas DG $110.02 \mathrm{VS}$, posicionadas a 0,5 m das plantas e com consumo de calda de $200 \mathrm{~L} \mathrm{ha}^{-1}$. As condições climáticas, registradas através de anemômetro digital portátil no momento das aplicações, foram em médias de $33{ }^{\circ} \mathrm{C}, 72 \%$ e $0,7 \mathrm{~km} \mathrm{~h}^{-1}$, respectivamente para temperatura, umidade relativa do ar e ventos. $\mathrm{O}$ curauá encontrava-se com 8 a 12 folhas e as plantas daninhas em início do desenvolvimento vegetativo, sendo a composição florística da área experimental, constituídas, em média, por 83,$21 ; 11 ; 6 ; 4 ; 4$ e 4 plantas $\mathrm{m}^{-2}$, respectivamente, de Cyperus rotundus (tiririca), Panicum maximum (capim-colonião), Boerhaavia diffusa (beldroega grande), Eleusine indica (capim-pé-de-galinha); Digitaria horizontais (capim colchão); Phyllanthus tenellus (quebra-pedra) e Sida spp. (guanxuma). No tratamento testemunha capinada, as plantas daninhas foram semanalmente controladas através de capinas manuais. A partir dos 30 DAA (Dias Após Aplicação), todos os tratamentos, com exceção da testemunha sem capina também tiveram a infestação semanalmente controlada por capinas manuais.

As características avaliadas foram: fitointoxicação (\%) aos 10, 20, 30, 40, 50 e 60 DAA (Dias Após Aplicação), através de notas visuais onde $0 \%$ corresponde à ausência de injúrias e $100 \%$ à morte das plantas de curauá (SBCPD, 1995); controle das plantas daninhas (\%) aos 10,20 e 30 DAA; altura média das folhas "D" e "E" de 5 plantas aos 10, 40, 70, 100 DAA e na pré-colheita, assim como número total de folhas/planta, número folhas beneficiáveis/planta na pré-colheita, produtividade de folhas totais e beneficiáveis (kg ha ${ }^{-1}$. De forma semelhante a do abacaxizeiro, as folhas $\mathrm{D}$ e $\mathrm{E}$ são as mais jovens entre as folhas adultas, e além de serem as mais compridas e ativas fisiologicamente, também são as utilizadas para avaliação de teores nutricionais (Cunha et al., 1999; 2002).

Os dados foram submetidos à análise de variância pelo teste $\mathrm{F}$ e as suas médias comparadas pelo teste de tukey ao nível de $5 \%$ de probabilidade. Para o controle da infestação e produtividade de folhas beneficiáveis, os dados originais médios foram ajustados aos modelos de regressão quadrático e linear.

\section{Resultados e discussão}

As plantas de curauá branco apresentaram boa tolerância em relação aos sintomas de fitointoxicação provocados pelo herbicida glyphosate, caracterizados por clorose e necrosamento de algumas pontas das folhas, uma vez que não foram constatados níveis de danos superiores a $22 \%$, a partir dos 10 DAA, entre as subdoses estudadas (Figura 1). A ação de glyphosate nas concentrações inferiores a 90,0 g e.a. ha ${ }^{-1}$ apresentaram sintomas mínimos de fitointoxicação visual, que podem ser facilmente confundidos com deficiências nutricionais ou condições de 
estresse hídrico, onde as folhas apresentam pontas e bordas em início de necrosamento. cloração mais amareladas e com algumas

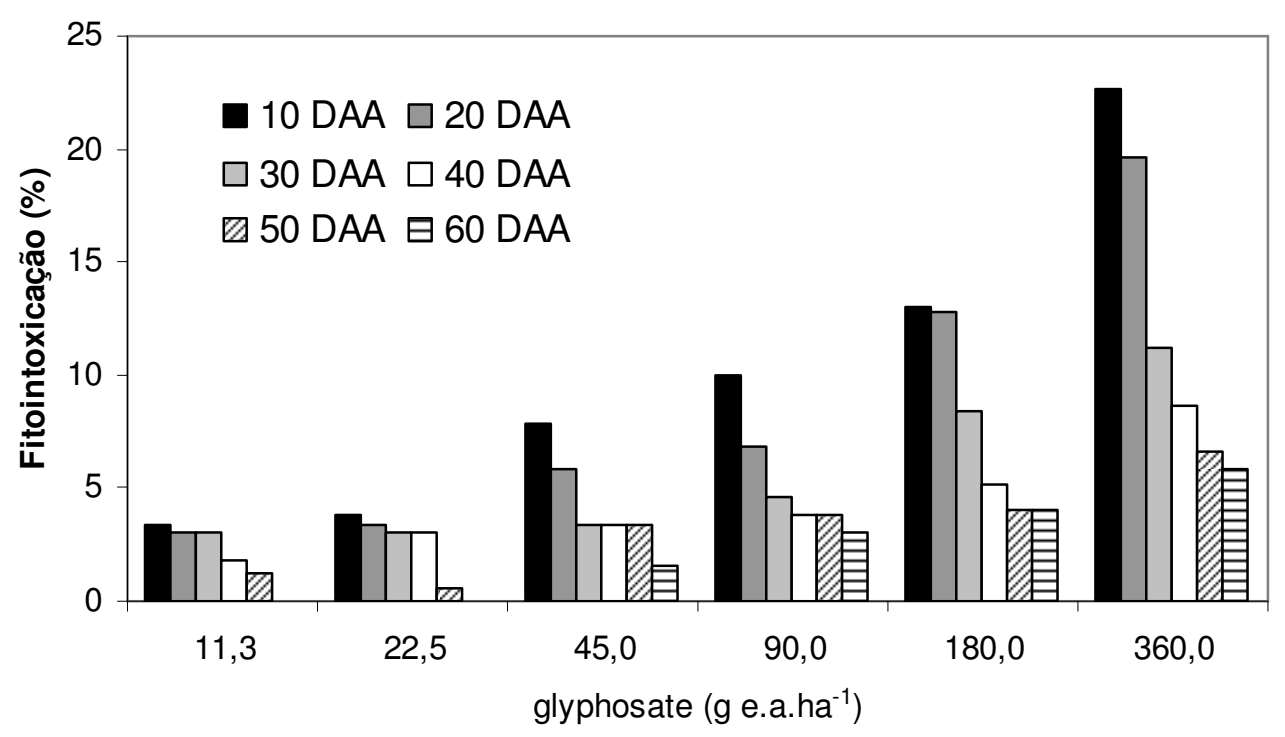

Figura 1. Fitointoxicação (\%) em plantas do curauá branco aos 10, 20, 30, 40, 50 e 60 DAA, submetidas à aplicação de subdoses de glyphosate.

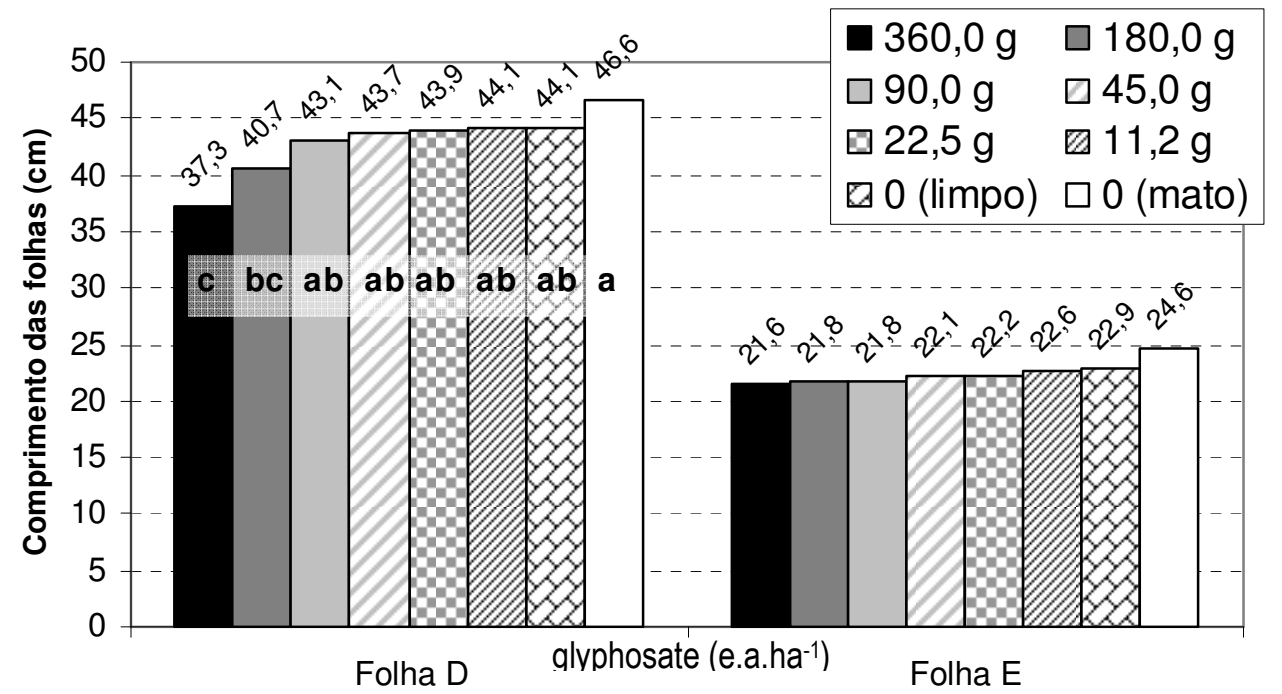

Figura 2. Comprimento das folhas da posição D e E (cm) de curauá branco aos 100 DAA, submetidas à aplicação de subdoses de glyphosate. 
Para o crescimento vegetativo do curauá branco (Figura 2) aos 100 DAA, não foram constatadas diferenças significativas para $\mathrm{o}$ comprimento das folhas da posição "E" entre as subdoses estudadas, ao contrário das folhas na posição "D", considerada com as folhas mais jovens e fisiologicamente ativas entre as demais (PY, 1969), onde apenas as subdoses inferiores a 90,0 $\mathrm{g}$ e.a.ha ${ }^{-1}$ não diferiram significativamente entre si, ou à testemunha capinada. As folhas das plantas de curauá nas posições $\mathrm{D}$ e $\mathrm{E}$, submetidas à convivência com a infestação até os 100 DAA apresentaram maior comprimento em função do possível estiolamento das plantas. Entretanto, com relação à característica número de folhas totais (Figura 3), as subdoses de glyphosate inferiores a 90,0 g e.a.ha ${ }^{-1}$ também não causaram diferenças significativas no desenvolvimento do curauá branco, ao contrário do número de folhas beneficiáveis, onde somente a concentração de 11,2 g e.a. ha ${ }^{-1}$ igualou-se a testemunha capinada. É importante ressaltar, que a convivência com a infestação de plantas daninhas durante todo o período reduziu significativamente o número de folhas totais e beneficiáveis do curauá branco, sendo essa interferência em torno de $38,1 \%$ e $55,2 \%$, respectivamente.

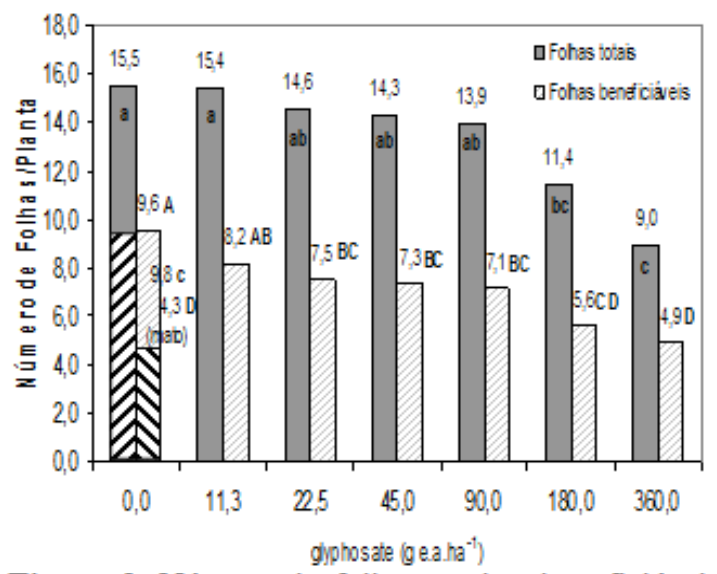

Figura-3. - Número-de-folhas totais-e-beneficiáveis por- planta-em- curauá branco- na- pré-colheita,
A produtividade de folhas beneficiais apresentou redução linear com o aumento das subdoses de glyphosate, sendo que apenas a concentração de 11,2 g e.a.ha ${ }^{-1}$ igualou-se significativamente a testemunha capinada (Figura 4), mas ainda caracterizando uma diferença média de $2380 \mathrm{~kg} \mathrm{ha}^{-1}(18,0 \%)$. Em termos de viabilidade econômica ou interação com o uso inadequado do glyphosate na cultura do curauá branco por problemas de deriva em aplicação de jato dirigido por exemplo, foi possível constatar que a tolerância da espécie ao glyphosate é baixa, visto que concentrações superiores a 45,0 g e.a.ha ${ }^{-1}$ promoveram as reduções mais significativas de produtividade. Estes resultados corroboram com os encontrados por Magalhães et al. (2001a; 2001b), onde baixas concentrações de glyphosate $\left(28,8\right.$ e 57,6 g e.a. ha $\left.{ }^{-1}\right)$ não afetaram o desenvolvimento de plantas de milho e sorgo, e diferiram dos relatados por Velini et al. (2008) e Cedergreen (2008), não sendo observados estímulos de crescimento do curauá branco para nenhuma das concentrações de glyphosate estudadas, mesmo para as subdoses inferiores a 36,0 g e.a.ha ${ }^{-1}$.

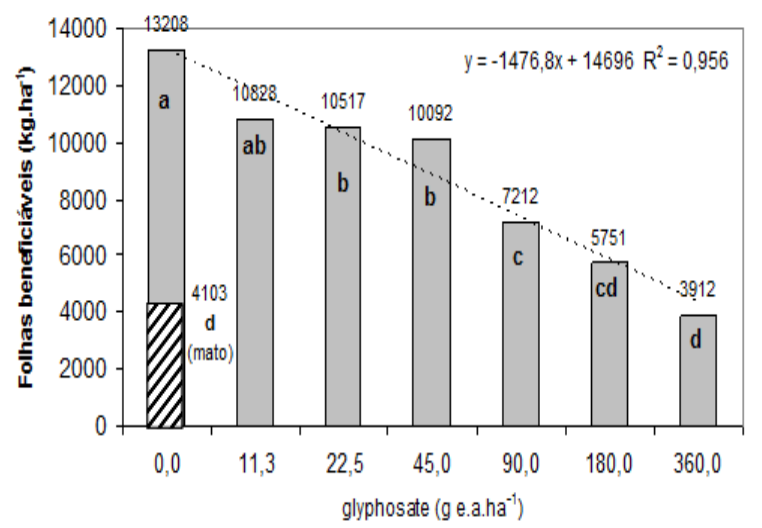

Figura 4. Produtividade de folhas beneficiáveis em plantas de curauá branco, submetidas à aplicação de subdoses de glyphosate.

Para o controle da infestação de plantas daninhas (Figura 5), foram constatados níveis satisfatórios (80\%) a partir das subdoses aplicadas nas concentrações de glyphosate 
superiores a 180,0 g e.a.ha ${ }^{-1}$, o que em contrapartida, interferiram negativamente no crescimento e produtividade de folhas beneficiáveis das plantas de curauá branco. Lacerda \& Victoria Filho (2004), determinando curvas de doses-resposta ao glyphosate (RC50), constataram que para reduzir $50 \%$ do crescimento das espécies Tridax procumbens, Digitaria insularis, Spermacoce latifolia, Ipomoea grandifolia, Commelina benghalensis necessitam de 58,40; 128,50; 250,44; 615,49 e $>1.440,0$ g e.a. ha $^{-1}$, respectivamente.

\section{Conclusões}

Nas condições em que foi realizado o trabalho, pode-se concluir que a espécie curauá branco (A. erectifolius) apresentou baixa tolerância ao herbicida glyphosate, sendo que subdoses superiores a 45,0 g e.a. ha $^{-1}$ promoveram reduções mais significativas de produtividade de folhas beneficiáveis. Nenhuma das subdoses estudadas promoveu estimulo ou incremento do desenvolvimento vegetativo das plantas de curauá branco.

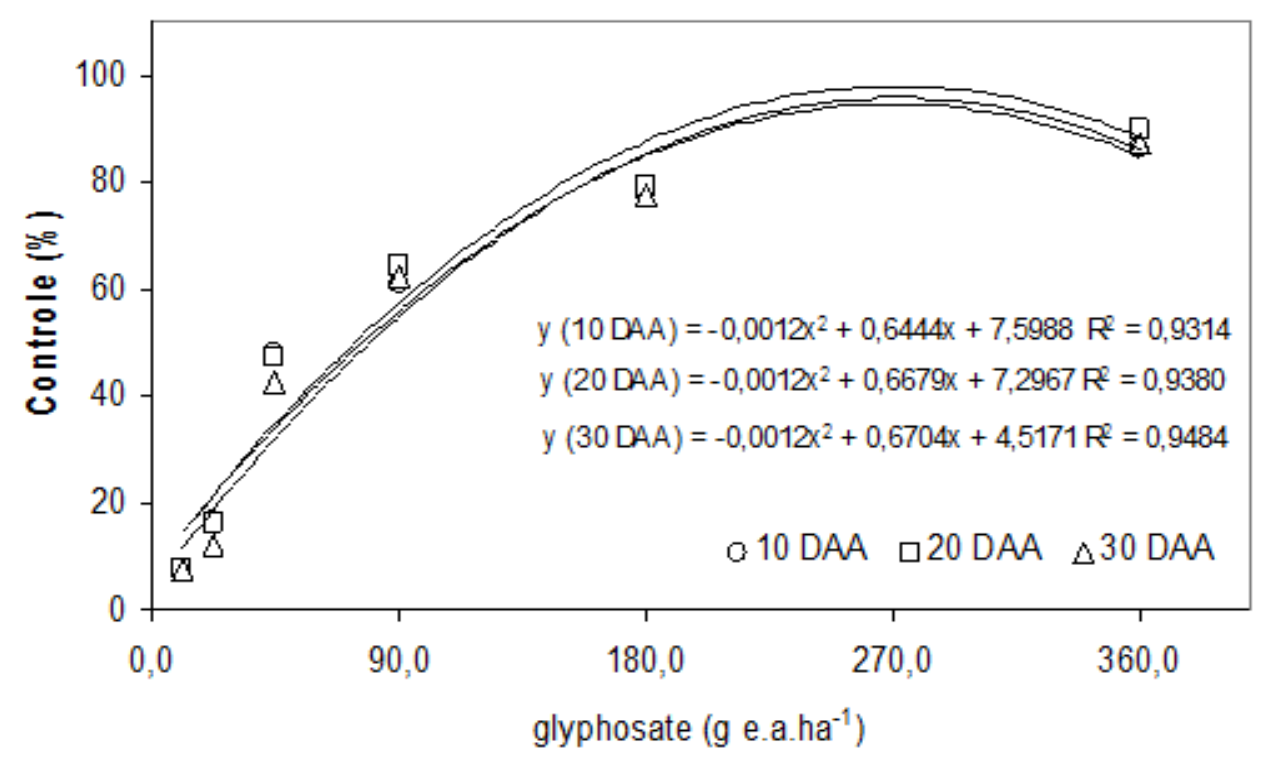

Figura 5. Controle (\%) de plantas daninhas na cultura do curauá branco aos 10 , 20 e 30 DAA, através de subdoses de glyphosate. 


\section{Agradecimento}

Ao apoio financeiro da empresa PEMATEC Triangel do Brasil Ltda para $\mathrm{O}$ desenvolvimento do projeto.

\section{Referências}

CARASCHI, J.C.; LEÃO, A.L. Mechanical Properties of Curauá Fiber Reinforced Polypropylene Composites. In: MATTOSO, L.H.C.; LEÃO, A.L e FROLLINI, E. Eds., Natural Polymers and Composites. São Carlos: Embrapa Instrumentação Agropecuária, 2000. p. 450-453.

CEDERGREEN, N. et al. The occurrence of hormesis in plants and algae. Dose-Response v.5, n.2, p.150-162, 2007.

CEDERGREEN, N. Herbicides can stimulate plant growth. Weed Research, v.48, n.5, p.429-438, 2008.

CUNHA, G.A.P. et al. O abacaxizeiro: cultivo, agroindústria e economia. Brasília: EMBRAPA, 1999. 480 p.

CUNHA, G. A. P.; COSTA, J. T. A.; REINHARDT, D. H. Alterações na massa foliar, produção de mudas, rendimento e colheita do abacaxizeiro "Pérola" causadas por reguladores de crescimento. Magistra, v. 14, n. 2, p.15-19, 2002.

DUKE, S.O.; CEDERGREEN, N.; VELINI, E.D.; BELZ, R.G. Hormesis: is it an important factor in herbicide use and allelopathy? Outlooks Pest Manag, v.17, n.1, p.29-33, 2006.

LACERDA, A.L.S.; VICTORIA FILHO, R. Curvas dose-resposta em espécies de plantas daninhas com o uso do herbicida glyphosate. Bragantia, v.63, n.1, p.73-79, 2004.

MAGALHÃES, et al. Efeito de doses reduzidas de glyphosate e paraquat simulando deriva na cultura do milho. Planta Daninha. v.19, n.2. p.247-253, 2001a.
MAGALHÃES et al. Efeito de doses reduzidas de glyphosate e paraquat simulando deriva na cultura do sorgo. Planta Daninha. v.19, n.2. p.255-262. 2001 b.

NEVES, D. C. et al. Hormese no crescmento do algodoeiro por subdoses de glifosato. In: CONGRESSO BRASILEIRO DO ALGODÃO, 7., 2009, Foz do Iguaçu. Sustentabilidade da cotonicultura Brasileira e Expansão dos Mercados: Anais... Campina grande: Embrapa Algodão, 2009. p.915-922.

PITELLI, R. A. Competição e controle de plantas daninhas em áreas agrícolas. IPEF, v.4, n.12, p. 25-35, 1987.

PY, C. La Piña Tropical. Barcelona: Editorial Blume, 1969. 278p.

REINHARDT, D.H.R.C.; CUNHA, G.A.P. Plantas daninhas e seu controle. MATOS, A.P. In: CUNHA, G.A.P. et al. (Org.). O abacaxizeiro: cultivo, agroindústria e economia. Brasília: Embrapa Mandioca e Fruticultura. 1 ed. 1999, p.253-268.

RODRIGUES, B.N.; ALMEIDA, F.S. Guia de Herbicidas. 5 ed. Londrina, PR, Ed. dos autores. 2005. 592p.

SCHABENBERGER， O.; KELLS， J. J.; PENNER, D. Statistical tests for hormesis and effective dosage in herbicide dose-response. Agronomy Journal, v.91, n.5, p.713-721, 1999.

SOCIEDADE BRASILEIRA DA CIÊNCIA DAS PLANTAS DANINHAS (SBCPD). Procedimentos para instalação, avaliação e análise de experimentos com herbicidas. Londrina: SBCPD, 1995. 42p.

TREZZI, M.M.; KRUSE, N.D.; VIDAL, R.A. Inibidores de EPSPs. In: Vidal, R.A. \& Meroto Jr., A. Herbicidologia. Porto Alegre, 2001. 152p.

WAGNER, R., KOGAN, M.; PARADA, A.M. Phytotoxic activity of root absorbed glyphosate in corn seedlings (Zea mays L.). 
Weed Biology and Management, v.3, n.4, p.228-232, 2003.

VELINI, E.D. et al. Glyphosate applied at low doses can stimulate plant growth. Pest Management Science, v.64, n.2, p.489-496, 2008.

VELINI, E.D. et al. Growth Regulation and Other Secondary Effects of Herbicides. Weed Science, v.58, n.3, p.351-354. 2010.

VIDAL, R.A. Herbicidas: mecanismos de ação e resistência de plantas. $1^{\mathrm{a}}$ ed. Porto Alegre: Edição dos Autores, 1997, 165p.

$4 \mathrm{p}$. 